Intramolecular Sensitization

DOI: 10.1002/anie.200504091

\section{Highly Efficient Photolabile Protecting Groups with Intramolecular Energy Transfer**}

\author{
Dominik Wöll, Julia Smirnova, Wolfgang Pfleiderer, \\ and Ulrich E. Steiner*
}

DNA chips are widely used for genomic analysis. ${ }^{[1]}$ Currently, high-density DNA chips can be synthesized with up to several hundred thousand different spots on an area of $1 \mathrm{~cm}^{2}{ }^{[2]} \mathrm{In}$

[*] D. Wöll, Dr. J. Smirnova, Prof. Dr. Dr. W. Pfleiderer, Prof. Dr. U. E. Steiner

Fachbereich Chemie, Universität Konstanz

Universitätstrasse 10, 78464 Konstanz (Germany)

Fax: $(+49) 7531-88-3014$

E-mail: ulrich.steiner@uni-konstanz.de

[***] We thank the Deutsche Forschungsgemeinschaft for financial support (project Ste 283/7-1) and NimbleGen GmbH, Waldkraiburg (Germany), for DNA chip experiments. principle, the complete human genome can be probed with such a chip. ${ }^{[3]}$ The required high spot densities can be achieved by photolithographic in situ DNA-chip synthesis. In this method, the oligonucleotides are synthesized step by step from protected nucleoside phosphitamides using photolithography with masks ${ }^{[4]}$ or micromirror arrays ${ }^{[5]}$ as suitable means for achieving parallel spatial addressability. This technique requires photolabile protecting groups ${ }^{[6]}$ of high light sensitivity that release their substrate (in this case a nucleotide) in nearly quantitative yield. For the protecting groups currently in use for DNA-chip synthesis, the light sensitivity, suitably characterized by the product $\varepsilon \phi$ of the absorption coefficient and the photochemical quantum yield, reaches only moderate values. The widely used [( $\alpha$-methyl-2-nitropiperonyl)oxy]carbonyl (MeNPOC) group $^{[7]}$ has a reasonable absorption coefficient at the wavelengths usually applied (in practice preferentially the mercury line at $\lambda=366 \mathrm{~nm}, \varepsilon_{\mathrm{MeOH}, 366 \mathrm{~nm}}$ $\approx 2500 \mathrm{M}^{-1} \mathrm{~cm}^{-1}$ ), but the photochemical deprotection yield is quite small $(3 \%$ in $\mathrm{MeOH}) .^{[8]}$ In contrast, the $2-(2-$ nitrophenyl)propoxycarbonyl (NPPOC) protecting group, ${ }^{[9]}$ the reaction of which is represented in Scheme $1,{ }^{[10]}$ shows a
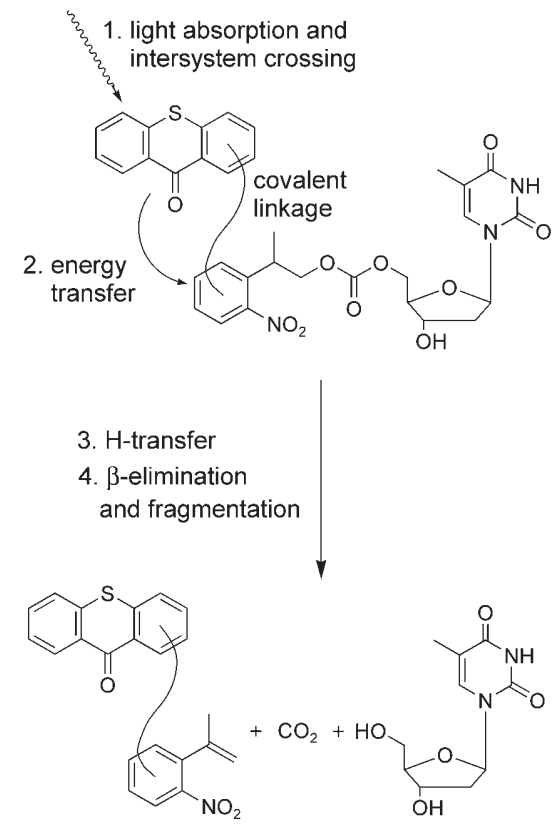

Scheme 1. Intramolecular sensitized photocleavage of a protecting group of the NPPOC type.

much higher quantum yield $(41 \%$ in $\mathrm{MeOH})$, but has a significantly lower absorptivity $\left(\varepsilon_{\mathrm{MeOH}, 366 \mathrm{~nm}} \approx 230 \mathrm{M}^{-1} \mathrm{~cm}^{-1}\right)$. Thus, for both MeNPOC and NPPOC fairly long irradiation times are required during which undesired photoreactions may take place.

Sensitization is a suitable method to improve the light sensitivity of weakly absorbing photolabile protecting groups. ${ }^{[11,12]}$ By using the triplet sensitizer thioxanthone to sensitize the photocleavage of NPPOC it has been possible to significantly improve the rate of photodeprotection in homogeneous solution as well as on a chip. ${ }^{[1]}$ In this case, most of the light energy is absorbed by the sensitizer and can be 
transferred to the protecting group if an encounter with a triplet-excited sensitizer molecule takes place during the lifetime of the latter. Diffusion is the limiting factor for the effectiveness of energy transfer under such intermolecular sensitization conditions. Various examples of intramolecular triplet-triplet energy transfer have been described. ${ }^{[13]}$

In this communication, we report the development of novel photolabile protecting groups wherein a diffusionindependent enhancement of light sensitivity is achieved by covalently linking the protecting group to a sensitizer chromophore. ${ }^{[14]}$ Recently the enhancement of photolysis of a photolabile protecting group by a covalently linked antenna molecule was also reported by the group of Corrie. ${ }^{[15]}$

The syntheses of the compounds shown in Scheme 2 have been described. ${ }^{[16]}$ Except for compound 7, the linkage of the

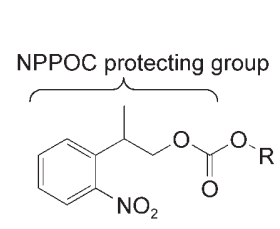

1

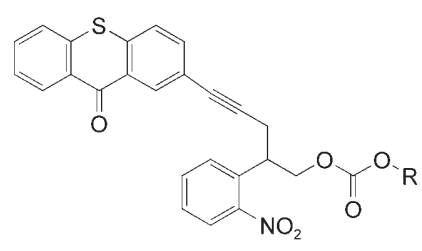

2

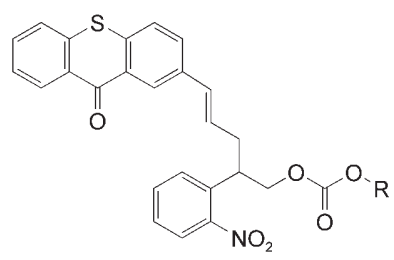

3

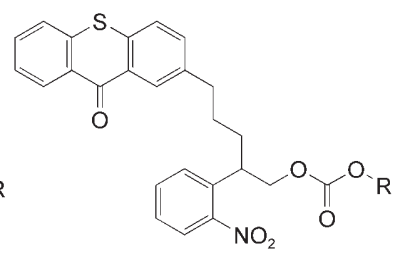

4
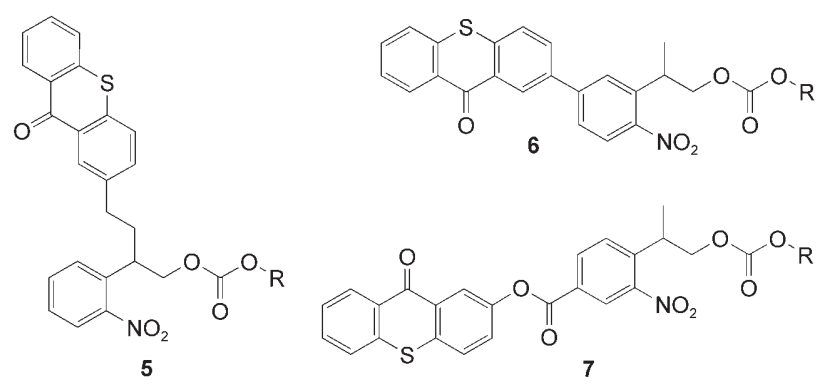

Scheme 2. Overview of the discussed compounds. $R=5^{\prime}$-O-thymidinyl.

$o$-nitrophenyl and thioxanthone chromophores at different positions and with different linkers was achieved by $\mathrm{C}-\mathrm{C}$ coupling reactions (Sonogashira, Heck, Suzuki). For synthesizing the protected thymidines $\left(\mathrm{R}=5^{\prime}\right.$ - $O$-thymidinyl $)$ connected to the protecting group by means of a carbonate bridge, the free alcohols $(\mathrm{R}=\mathrm{H})$ were first treated with phosgene or phosgene substitutes and then with thymidine.

Figure 1 shows the absorption spectra of the protected thymidines. Except for compound 6, the absorptions are essentially determined by the thioxanthone moiety. Significant spectral changes in the substituted thioxanthones appear in the second absorption band for the 2-vinyl- and the 2ethynyl-substituted derivatives. The NPPOC chromophore

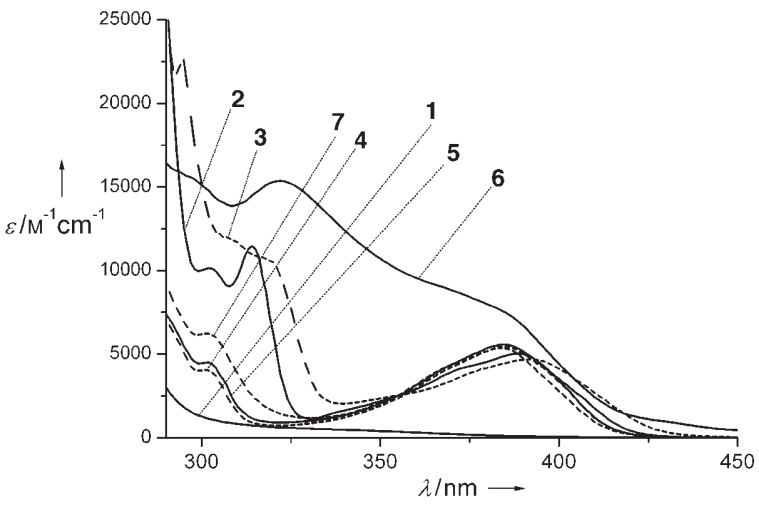

Figure 1. Absorption spectra of compounds $\mathbf{1 - 7}$ in methanol.

contributes only a fairly weak absorption; the thymidine moiety does not absorb at all in the spectral range shown. Compound $\mathbf{6}$ is the only one exhibiting a direct electronic coupling between the two chromophores through the $\pi$ electron system. As a result of the thioxanthone moiety the bichromophoric protecting groups 2-5 and $\mathbf{7}$ exhibit absorption coefficients at $366 \mathrm{~nm} 15$ times higher, and compound $\mathbf{6}$ even about 35 times higher, than that of the NPPOC protecting group 1. Thus, for unchanged photochemical quantum yield, an approximately 15 or 35 times higher light sensitivity would result. To test this expectation, the thymidines capped with the new protecting groups were subject to continuous irradiation for defined periods of time (conditions: 0.05 and $0.15 \mathrm{~mm}$ in methanol, photon irradiance about $2 \times 10^{-8}$ Einstein $\mathrm{cm}^{-2} \mathrm{~s}^{-1}$ as determined by azobenzene actinometry $\left.{ }^{[17]}\right)$. The products were separated by HPLC and analyzed by UV/Vis spectrometry. The decay kinetics of the starting compound is described by the rate law given in Equation (1). Here $I_{0}$ represents the photon irradiance, $F$ the

$\frac{d c}{d t}=-I_{0} \frac{F d}{V} \frac{\left(1-10^{-A(t)}\right)}{A(t)} \varepsilon c(t) \phi$

illuminated cross section, $d$ the optical path length, $V$ the volume of the solution, $A(t)$ the absorbance of the solution after irradiation time $t, \varepsilon$ the molar absorption coefficient of the protected compound, $c$ its concentration, and $\phi$ the quantum yield of the photodeprotection reaction. The quantum yield $\phi$ is determined by fitting the result $c(t)$ of the numerical integration of Equation (1) to the observed time dependence (Figure 2a).

The data in Table 1 indicate that the quantum yields of the bichromophoric protecting groups are somewhat lower than that of the NPPOC protecting group. However, this reduction of the quantum yield is overcompensated by the increased absorption coefficient such that the resulting overall enhancement of light sensitivity reaches a factor of up to 21 at $366 \mathrm{~nm}$ and 25 at $405 \mathrm{~nm}$. For compounds 4-7 the yields of deprotected substrate range from 66 to $92 \%$ (Table 1). A further improvement of these values by systematic optimization of the reaction conditions should be possible. The poor thymidine yield of compounds $\mathbf{2}$ and $\mathbf{3}$ is a result of specific photochemical side reactions. Whereas the major reaction of 

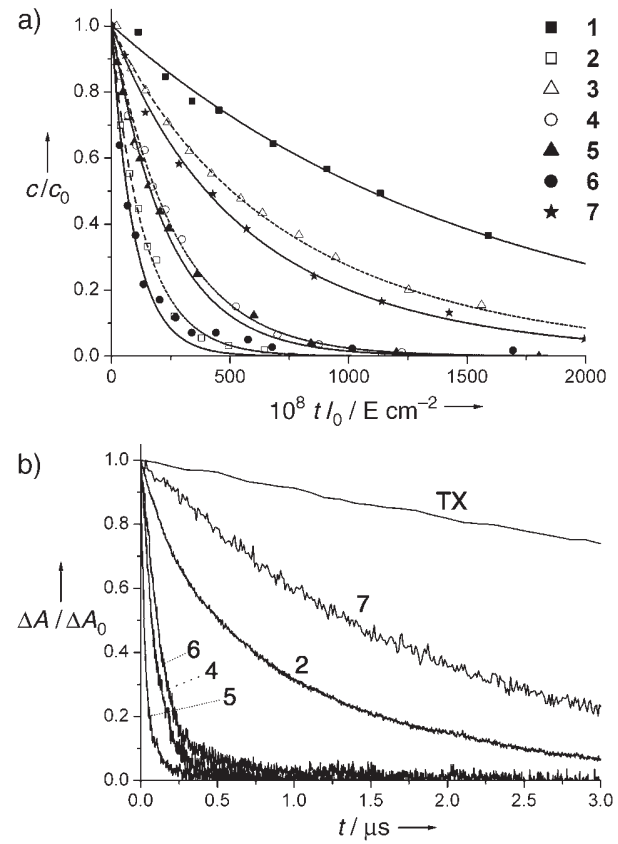

Figure 2. a) Decomposition kinetics of the protected thymidines 1-7 when continuously irradiated at $\lambda=366 \mathrm{~nm}$. The photon irradiance $I_{0}$ was on the order of $2 \times 10^{-8}$ Einstein $\mathrm{cm}^{-2} \mathrm{~s}^{-1}$. Data points were calculated by integration of the HPLC peaks observed after the corresponding irradiation times. Curves were fitted to the data points according to Equation (1). b) Decay kinetics of the thioxanthone triplet observed by transient absorption at $600 \mathrm{~nm}$ after ns laser flash photolysis (excitation at $355 \mathrm{~nm}$ ). No triplet signal could be observed for compound 3.

compound $\mathbf{3}$ is a trans-cis isomerization, the main product from compound $\mathbf{2}$ is a rearrangement product of identical molar mass but with an as-yet unidentified structure.

Compounds 4 and 6 have already been tested for DNAchip synthesis. Under optimized conditions, the cycle times could be reduced by a factor of 10 compared to the reaction time with NPPOC-T (compound $\mathbf{1}, \mathrm{R}=5^{\prime}$ - $O$-thymidinyl). The yield per synthesis cycle was about $90 \%$.

To prove that the increased light sensitivity is a result of an intramolecular triplet energy transfer, nanosecond laser flash spectroscopic experiments were performed. Transient absorption spectra as well as decay curves of the thioxanthone triplet were recorded. Following the change of the transient absorption spectra in time, the triplet-triplet absorption decay as well as the formation of the aci-nitro form of the NPPOC group, a known intermediate of the deprotection step of NPPOC ${ }^{[10]}$ could be detected.

In $\mathrm{N}_{2}$-saturated solution, the thioxanthone triplet lifetimes for all bichromophoric compounds are much shorter than for unmodified thioxanthone (see Figure 2 and Table 1). The combination of this finding and the similarity of the quantum yields of sensitized and directly excited photoreaction of NPPOC proves that triplet quenching arises mainly from energy transfer. Not only the ground-state absorption spectrum of compound 6 (see Figure 1) but also its excited-state absorption spectrum differs significantly from the corresponding ones obtained for the other linked thioxanthones. In 6, the two chromophores are strongly coupled and therefore cannot be regarded as independent. In this system, the lifetime of the observed transient is presumably determined by the intramolecular $\mathrm{H}$-transfer step initiating the photoreaction. The photochemical quantum yield of $\mathbf{6}$ is comparable to that of the other linked thioxanthone-NPPOC conjugates connected by longer links with less direct coupling.

In aerated solution, molecular oxygen acts as triplet quencher in a close-to-diffusion-controlled reaction and reduces the triplet lifetime of all thioxanthone compounds to a value in the $100-n s$ range. Since this process competes with energy transfer it reduces the quantum yield of the photocleavage. If intramolecular energy transfer is fast or, as in compound $\mathbf{6}$, if the lifetime of the triplet is short because of its fast reaction, the light sensitivity of the photoreaction depends much less on the oxygen content in solution than with a free triplet sensitizer. Therefore, the photokinetics of compounds 4-6 are not very sensitive to the presence of oxygen. This trend could also be demonstrated for the new protecting groups under conditions of continuous illumination in aerated solution. The reduced sensitivity towards oxygen is an important advantage for their application in the industrial production of DNA chips because an oxygen-free process would be technically more demanding.

Although the involvement of triplet-triplet energy transfer has been clearly demonstrated for the new compounds, it must be noted that sensitization of the photocleavage reaction cannot be explained exclusively on the basis of this mechanism. Such a conclusion follows from the observation of considerable fluorescence quenching, particularly in the cases with the shortest linkers, which is paralleled by a correspondingly reduced amount of triplet formation, but which does not

Table 1: Spectroscopic and photochemical data for compounds 1-7..$^{[a]}$

\begin{tabular}{|c|c|c|c|c|c|c|c|c|c|c|}
\hline \multirow[b]{2}{*}{ Cmpd. } & \multicolumn{2}{|c|}{$\varepsilon\left[\mathrm{M}^{-1} \mathrm{~cm}^{-1}\right]$} & \multicolumn{2}{|c|}{$\phi$} & \multicolumn{2}{|c|}{$\varepsilon \phi\left[\mathrm{M}^{-1} \mathrm{~cm}^{-1}\right]$} & \multicolumn{2}{|c|}{ Thymidine yield } & \multicolumn{2}{|c|}{$\tau[\mu \mathrm{s}]$} \\
\hline & $366 \mathrm{~nm}$ & $405 \mathrm{~nm}$ & $366 \mathrm{~nm}$ & $405 \mathrm{~nm}$ & $366 \mathrm{~nm}$ & $405 \mathrm{~nm}$ & $366 \mathrm{~nm}$ & $405 \mathrm{~nm}$ & $\mathrm{~N}_{2}$-sat. & Air-sat. \\
\hline 1 & 250 & $<40$ & 0.41 & - & 100 & $<40$ & 0.90 & - & [b] & [b] \\
\hline 2 & 3500 & 2700 & 0.42 & 0.38 & 1500 & 1000 & 0.21 & 0.33 & 1.05 & 0.18 \\
\hline 3 & 3100 & 3400 & 0.08 & 0.05 & 250 & 170 & 0.27 & 0.38 & {$[c]$} & [c] \\
\hline 4 & 3800 & 2300 & 0.21 & 0.14 & 800 & 320 & 0.86 & 0.75 & 0.11 & 0.08 \\
\hline 5 & 3900 & 2300 & 0.21 & 0.14 & 820 & 320 & 0.75 & 0.79 & 0.04 & 0.03 \\
\hline 6 & 8200 & 2400 & 0.26 & 0.27 & 2100 & 650 & 0.66 & 0.89 & 0.13 & 0.12 \\
\hline 7 & 4000 & 1800 & 0.09 & 0.09 & 360 & 160 & 0.92 & 0.82 & 3.6 & 0.22 \\
\hline
\end{tabular}

[a] Molar decadic absorption coefficient $\varepsilon$, photochemical quantum yield $\phi$, light sensitivity $\varepsilon \phi$, deprotected thymidine yield, and triplet lifetime $\tau$. [b] For unsubstituted thioxanthone the triplet lifetime is $22.2 \mu \mathrm{s}$ in $\mathrm{N}_{2}$-saturated $\mathrm{MeOH}$ and $0.18 \mu$ s in air-saturated $\mathrm{MeOH}$. [c] $\mathrm{No}$ transient with a lifetime in the nanosecond time range could be detected. 
go along with a decrease of the overall photochemical quantum yield. Detailed investigations to clarify the underlying mechanism are in progress.

In summary, a series of novel, highly light-sensitive photolabile protecting groups for light-controlled DNA synthesis has been developed. In these compounds the NPPOC protecting group is covalently linked to thioxanthone as an intramolecular antenna. The photochemical kinetics of these compounds under stationary irradiation conditions has been quantitatively investigated, and photochemical quantum yields as well as chemical yields of the photodeprotected substrate were determined for thymidine as a model substrate. The kinetics of triplet-triplet energy transfer between the antenna molecule and the photolabile protecting group has been investigated by laser flash spectroscopy. Besides triplet-triplet energy transfer, a sensitization mechanism involving the excited sensitizer singlet must be also involved, particularly in the systems with short linkers. As a result of the high light sensitivity of these new protecting groups, it should be possible to reduce the production time for the photolithographic synthesis of high-density DNA chips.

Received: November 17, 2005

Published online: March 23, 2006

Keywords: DNA chips - intramolecular energy transfer . photochemistry $\cdot$ protecting groups $\cdot$ sensitization

[1] An excellent overview is given in the special issue Nat Genet. 1999, 21 (1), $1-60$.

[2] K.-P. Stengele, U. E. Steiner, D. Wöll, W. Pfleiderer, S. Bühler, J. Bühler, R. Green, Abstr. Pap. Solid Phase Synthesis \& Combinatorial Libraries, London, United Kingdom, 2003; http://www. biocom.uk/sp s2003.htm.

[3] NimbleGen Systems Inc., NimbleGen Releases First Human Whole-Genome Array, Pressemitteilung, Madison, WI, 28. Juli 2003; http://www.nimblegen.com/news/press_rel/news_2003 _07_28.html.

[4] S. P. A. Fodor, J. L. Read, M. C. Pirrung, L. Stryer, A. T. Lu, D. Solas, Science 1991, 251, 767-773.
[5] S. Singh-Gasson, R. D. Green, Y. Yue, C. Nelson, F. Blattner, M. R. Sussman, F. Cerrina, Nat. Biotechnol. 1999, 17, 974-978.

[6] "Photoremovable Protecting Groups in DNA Synthesis and Microarray Fabrication": M. C. Pirrung, V. S. Rana in Dynamic Studies in Biology: Phototriggers, Photoswitches, and Caged Compounds (Eds.: M. Goeldner, R. S. Givens), Wiley, New York, 2005, p. 341.

[7] a) G. H. McGall, A. D. Barone, M. Diggelmann, S. P. A. Fodor, E. Gentalen, N. Ngo, J. Am. Chem. Soc. 1997, 119, 5081-5090; b) A. C. Pease, D. Solas, E. J. Sullivan, M. T. Cronin, C. P. Holmes, S. P. A. Fodor, Proc. Natl. Acad. Sci. USA 1994, 91, $5022-5026$

[8] The quantum yield for MeNPOC was determined under identical condition as for NPPOC $(\mathrm{MeOH}$, irradiation at $366 \mathrm{~nm})$.

[9] a) A. Hasan, K.-P. Stengele, H. Giegrich, P. Cornwell, K. R. Isham, R. A. Sachleben, W. Pfleiderer, R. S. Foote, Tetrahedron 1997, 53, 4247-4264; b) H. Giegrich, S. Eisele-Bühler, C. Hermann, E. Kvasyuk, R. Charubala, W. Pfleiderer, Nucleosides Nucleotides 1998, 17, 1987-1996; c) S. Bühler, I. Lagoya, H. Giegrich, K.-P. Stengele, W. Pfleiderer, Helv. Chim. Acta 2004, $87,620-659$.

[10] S. Walbert, W. Pfleiderer, U. E. Steiner, Helv. Chim. Acta 2001, $84,1601-1611$.

[11] D. Wöll, S. Walbert, K.-P. Stengele, T. J. Albert, T. Richmond, J. Norton, M. Singer, R. D. Green, W. Pfleiderer, U. E. Steiner, Helv. Chim. Acta 2004, 87, 28-45.

[12] a) C. Sundararajan, D. E. Falvey, J. Am. Chem. Soc. 2005, 127, $8000-8001$; b) C. Sundararajan, D. E. Falvey, Org. Lett. 2005, 7 , 2631-2634; c) A. Banerjee, K. Lee, D. E. Falvey, Tetrahedron 1999, 55, 12699-12710.

[13] S. Speiser, Chem. Rev. 1996, 96, 1953-1976 and references therein.

[14] U. E. Steiner, Universität Konstanz, D. Wöll, DE 10315772 (A1), WO 2004089529, 2004 [Chem. Abstr. 2004, 141, 366034].

[15] G. Papageorgiou, M. Lukeman, P. Wan, J. E. T. Corrie, Photochem. Photobiol. Sci. 2004, 3, 366-373; G. Papageorgiou, D. Ogden, J. E. T. Corrie, J. Org. Chem. 2004, 69, 7228-7233.

[16] J. Smirnova, D. Wöll, W. Pfleiderer, U. E. Steiner, Helv. Chim. Acta 2005, 88, 891-904.

[17] G. Gauglitz, J. Photochem. 1976, 5, 41-47; G. Gauglitz, S. Hubig, J. Photochem. 1981, 15, 255-257. 